\title{
Interaction matrix uncertainty in active (and adaptive) optics
}

\author{
Douglas G. MacMynowski \\ Control and Dynamical Systems, California Institute of Technology, 1200 East California Boulevard, \\ Pasadena, California 91125, USA (macmardg@ cds.caltech.edu)
}

Received 10 December 2008; revised 13 March 2009; accepted 20 March 2009; posted 20 March 2009 (Doc. ID 105029); published 3 April 2009

\begin{abstract}
Uncertainty in the interaction matrix between sensors and actuators can lead to performance degradation or instability in control of segmented mirrors (typically the telescope primary). The interaction matrix is ill conditioned, and thus the position estimate required for control can be highly sensitive to small errors in knowledge of the matrix, due to uncertainty or temporal variations. The robustness to different types of uncertainty is bounded here using the small gain theorem and structured singular values. The control is quite robust to moderate uncertainty in actuator gain, sensor gain, or the ratio of sensor dihedral and height sensitivity. However, the control is extremely sensitive to small errors in geometry, with the maximum error that can be tolerated scaling inversely with the number of segments. The same tools can be applied to adaptive optics; however, the interaction matrix here is better conditioned and so uncertainty is less of an issue, with the tolerable error scaling inversely with the square root of the number of actuators. (C) 2009 Optical Society of America
\end{abstract}

OCIS codes: $110.6770,220.1080$.

\section{Introduction}

Active optics (control of telescope mirrors) and adaptive optics (compensating for atmospheric turbulence with a deformable mirror) both require knowledge of the interaction matrix (IM) that relates the displacement at actuator locations to the resulting sensor response. At existing observatories, robustness to errors in this matrix has not been an issue. However, robustness may become an issue for future larger telescopes as the number of degrees of freedom in the interaction matrices increases. This is particularly relevant in the control of highly segmented primary mirrors planned for large ground-based optical telescopes where 492 and 984 segment mirrors are being designed [1,2]. However, the approach and general conclusions can also be applied to other active optics or adaptive optics (AO) control problems; to illustrate this, analysis is also included for a singleconjugate AO problem.

0003-6935/09/112105-10\$15.00/0

(C) 2009 Optical Society of America
For segmented-mirror control, the three out-ofplane degrees of freedom of each segment are controlled using actuators behind the segments. The positions of the segments are estimated using relative measurements between neighboring segments. In general, these measurements are sensitive to both relative segment out-of-plane displacement and also the dihedral angle between segments. The sensor response to segment displacement can be described with an interaction matrix $A$ (see Chanan et al. [3]). This matrix is ill conditioned, and thus the estimate of the segment displacement is highly sensitive to certain types of uncertainty in the interaction matrix. The same sensing and control approach is used successfully at existing telescopes [4]. However, the condition number of the interaction matrix increases as the number of segments increases, and hence robustness to uncertainty decreases.

AO control involves a similar estimation based on the interaction matrix between the residual (postcorrection) wavefront phase at the deformable mirror actuator locations and the corrected wavefront slope measurements (see [5]). The AO interaction matrix is typically reasonably well conditioned even for large 
problems, and thus uncertainty is less of a concern, although AO control has been shown to be sensitive to misregistration errors, e.g., [6]. Higher emphasis in the presentation is therefore given to the segmented-mirror control problem for which the analysis demonstrates that interaction-matrix uncertainty is a potential issue.

The analytical results apply to any quasi-static estimation problem, although illustrative quantitative examples are presented only for segmented-mirror control and single-conjugate AO, as these are the only two cases in which the number of degrees of freedom in the interaction matrices is expected to be large. The focus is on understanding what types of uncertainty are a potential problem, and illustrating tools that can be applied to any problem, rather than an exhaustive analysis of all possible types of uncertainty. Also note that the effects of uncertainty in a single scalar are straightforward to analyze by construction (e.g., single-axis misregistration for AO, or gravity-induced changes as a function of observing zenith angle for active optics). However, the potential interactions from multivariable uncertainty are less obvious.

Four types of uncertainty are considered herein. First, errors in actuator gain; these errors are the same for each column of the interaction matrix. Second and third, errors in sensor gain: either the overall gain, or (for segmented-mirror control) the ratio of sensitivity to displacement and to dihedral angle. These errors are the same for each row of the interaction matrix. And fourth, errors in geometry (e.g., the exact locations of each sensor) that can affect every nonzero element of the interaction matrix differently. Analysis is first conducted using the small gain theorem, e.g., [7,8]; a standard tool for computing robustness bound to unstructured uncertainty. This is sufficient to illustrate that the system is highly sensitive to only the last of these uncertainties. The robustness boundary for this case is obtained using structured singular values ( $\mu$ analysis) [9], which takes into account the specific form of the uncertainty. The analysis tools are applicable to any additional structure one might wish to place on the form of the uncertainty in the interaction matrix, e.g., to capture uncertainty in specific degrees of freedom in the location of actuators or sensors. The robustness boundary is not highly sensitive to the control bandwidth assumed.

Quantitative results are computed as a function of the number of segments for a segmented mirror, and as a function of the number of actuators for singleconjugate AO. Some additional detail is provided for a 492-segment primary mirror [the Thirty Meter Telescope (TMT) design], and for the 36-segment Keck primary mirror as comparison. The analytically computed bound demonstrates that it is possible to construct a hypothetical combination of errors in the interaction matrix that leads to instability with very small magnitude errors. A statistical analysis is also used to understand how likely it is that errors bounded by a given magnitude will result in instability.

The paper is organized as follows. Section 2 describes the interaction matrix for a segmented mirror and gives many of the properties of the matrix that will be useful in computing analytical robustness bounds. The analysis tools are presented in Section 3, starting first with small gain analysis that is sufficient to understand the actuator and sensor gain errors, and then structured singular value analysis specific to the geometry errors. Quantitative examples are given in Section $\underline{4}$ and for AO in Section $\underline{5}$.

\section{Problem Definition}

\section{A. Segmented-Mirror Control}

For existing and future optical telescopes with segmented primary (or other) mirrors, the segments are typically nearly hexagonal, with three actuators behind each segment to control the piston, tip, and tilt of the segment [4]. (The analytical results in Section 3 do not depend on the segmentation geometry, only the quantitative results in Section 4 do.) On each intersegment edge are two sensors that are in general sensitive to both the relative out-of-plane displacement of the segments and also the relative dihedral angle between segments. The segmentation geometry and actuator/sensor layout for the $N=492 \mathrm{seg}$ ment primary mirror of the TMT are shown in Figs. 1 and 2; for this geometry there are $n=1476$ actuators and $m=2772$ sensors. The control problem consists of two steps: first, estimating the segment displacement at the actuator locations, and then control of the segment displacement.

The relationship between the displacement $x \in \mathbb{R}^{n}$ of the mirror segments at the actuator positions and the sensor measurements $y \in \mathbb{R}^{m}$ can be described by

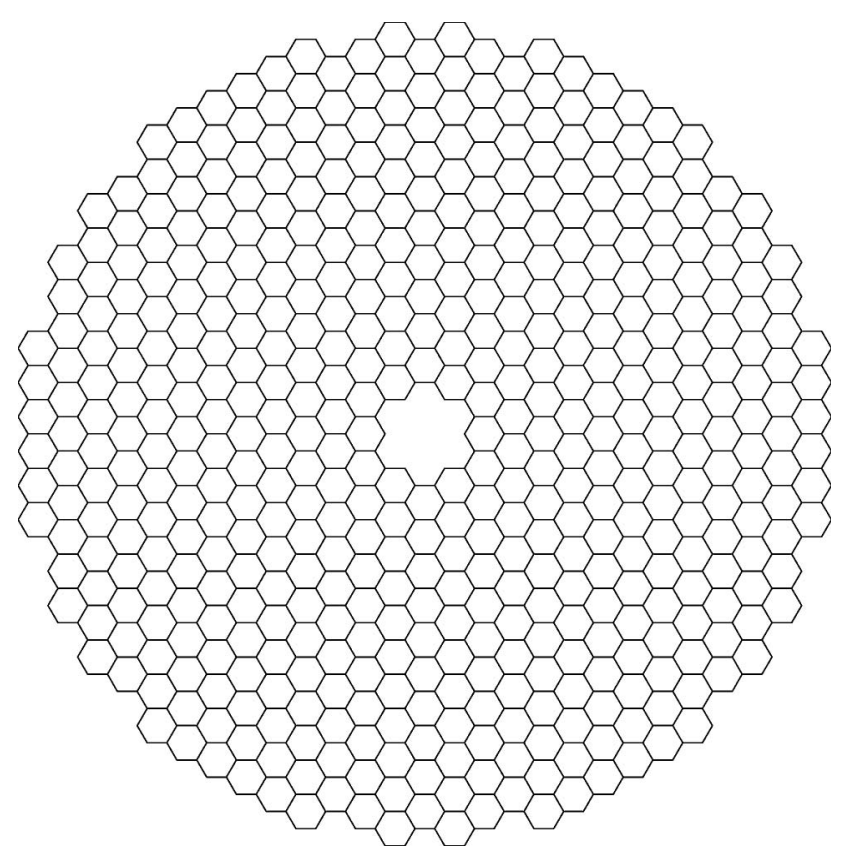

Fig. 1. Segmentation layout for a 492-segment mirror. 


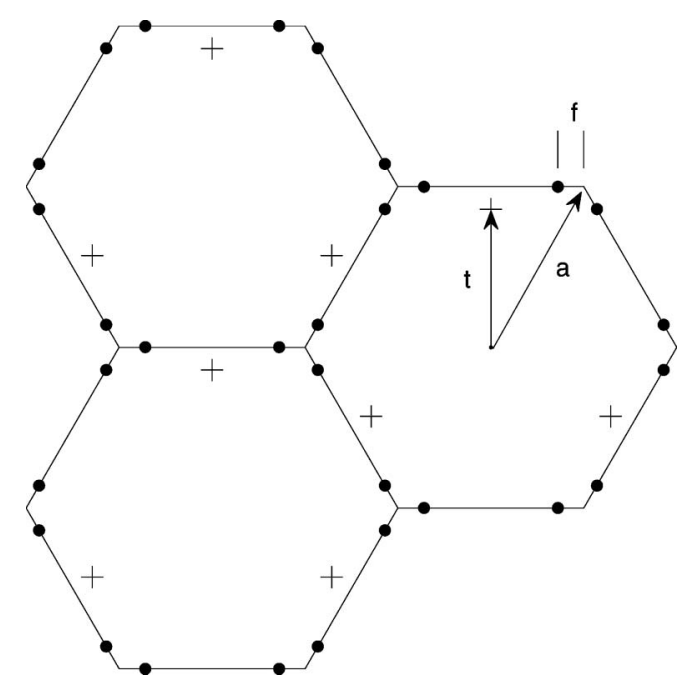

Fig. 2. Detail on three segments illustrating locations of actuator “+" and intersegment sensor "•". Parameters used in examples are segment radius $a=0.715 \mathrm{~m}$, actuator radius $t=0.742 a$, and distance from sensor to vertex $f=0.14 a$.

$$
y=A x
$$

In general, there is also a sensor offset that must be estimated using wavefront information; however, this can be subtracted from the sensor measurement and thus does not affect the feedback control problem. The propagation of sensor noise is understood $[3,10]$, and while this may limit the desired control bandwidths, it does not otherwise affect the feedback problem.

The interaction matrix $A \in \mathbb{R}^{m \times n}$ can be estimated from geometry [3]. The response of each sensor depends only on the positions of the neighboring segments, and hence each row of $A$ has only 6 nonzero entries. With any relative sensor measurement, the global piston, tip, and tilt of the mirror are unobservable, so $A^{T} A$ has rank $n-3$. With zero dihedral sensitivity, there is also a fourth unobservable "focus mode," where each segment tilts relative to its neighbors but with no relative height change between segments. This deflection pattern is primarily a change in the mirror radius of curvature and thus looks like a change in focus, with some additional scalloping in the wavefront because each segment radius of curvature has not changed. Nonzero dihedral sensitivity makes this mode observable, but with small sensitivity does not significantly affect the observability of other modes; this result will be important later in the paper.

A few additional observations on the structure of $A$ will be relevant in subsequent analysis.

The matrix $A$ can be expressed as the sum of the sensor displacement and dihedral sensitivity components as

$$
A=A_{h}+A_{\theta}
$$

The height sensitivity is dimensionless (the segment edge displacement at the sensor location per unit segment displacement at actuator location), and thus $A$ is dimensionless. The ratio of sensor dihedral sensitivity and height sensitivity (the effective sensor moment arm; see Fig. 3) is a design parameter with units of length, denoted here as $L_{\text {eff }}$ (parameter $-g$ or $\eta h$ in [3]). Referring to Eq. (2) or (9) in Chanan et al. [3], each sensor response to an actuator displacement is of the form $\alpha+L_{\text {eff }} \beta$; these two terms correspond to the two terms in Eq. (2). The entries in the $A$ matrix depend on the nondimensional parameter $L_{\text {eff }} / a$, as well as on the nondimensional geometry parameters $t / a$ and $f / a$ shown in Fig. 2 .

The sensitivity to focus mode, expressed as the corresponding singular value of $A$ [see Eq. (7)] scales roughly as $0.9\left(L_{\text {eff }} / a\right) N^{-1 / 2}$. The next smallest nonzero singular values, corresponding to astigmatism, scale roughly as $2.5 / N$; these scalings can both be verified by construction using the formulas in [3]; the astigmatism scaling also follows from Eq. (6) in [10]. Even for the relatively large sensor moment arm at Keck of $L_{\text {eff }}=55 \mathrm{~mm}$, focus mode will have the smaller sensitivity for any telescopes currently being designed. Sensor designs for TMT may have $L_{\text {eff }}$ as small as $10 \mathrm{~mm}$, and this value $\left(L_{\mathrm{eff}} / a=\right.$ 0.014 ) is used throughout for quantitative examples.

Each sensor is only influenced by $q=6$ actuators (on neighboring segments), and each actuator influences at most 12 sensors (two per intersegment edge in the Keck and TMT geometry). By separating out the influence of each of these gains separately, the $A$ matrix can also be written as

$$
A=\mathcal{P} \underline{A} \mathcal{R}
$$

where $\underline{A} \in \mathbb{R}^{q m \times q m}$ has all the nonzero elements of $A$ on its diagonal: if $A_{i j}=a_{k}$, then $\underline{A}_{k k}=a_{k}, \mathcal{P}_{i k}=1$, and $\mathcal{R}_{k j}=1$. This decomposition can be easily constructed and is not unique. One possible choice for the matrix $\mathcal{P}$ is

$$
\mathcal{P}=\left[\begin{array}{lll}
I_{m} & I_{m} & \ldots I_{m}
\end{array}\right]
$$

where the identity matrix $I_{m}$ of dimension $m$ is repeated $q=6$ times to add the contributions from the neighboring actuators (this implies a particular ordering of the nonzero elements of $A$ ). The matrix $\mathcal{R}$ is defined to separate the influence of each actuator on all possible sensors and retain the correct

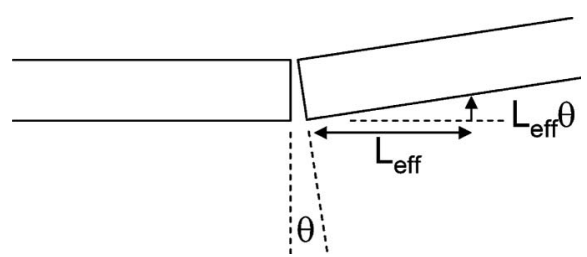

Fig. 3. Definition of sensor moment $\operatorname{arm} L_{\text {eff }}$; a sensor measuring relative height a distance $L_{\text {eff }}$ from the segment edge will give output $z=h+L_{\text {eff }} \theta$. At Keck, the dihedral sensitivity resulted from an actual moment arm; other sensor geometries give rise to dihedral sensitivity from other mechanisms but can still be characterized in terms of $L_{\text {eff }}$. 
actuator-sensor connectivity of $A$, with a nonobvious structure that depends on the segment and sensor numbering convention. This decomposition of $A$ will be useful in computing tight robustness bounds to uncertainty in Subsection 3.B.

The segment positions can be estimated from the sensor measurements. A least-squares estimate is typically sufficient, so $\hat{x}=A^{+} y$, where the pseudoinverse is

$$
A^{+}=\lim _{\epsilon \rightarrow 0}\left(A^{T} A+\epsilon I\right)^{-1} A^{T} .
$$

More general linear reconstructors $\hat{x}=B y$ can easily be incorporated in the analysis, but as less can be said about the completely general setting, I provide quantitative results only for $B=A^{+}$. Computationally efficient reconstructors (e.g., multigrid or Fourier methods) that converge to the least-squares solution will yield essentially the same result.

The pseudoinverse is typically computed from the singular value decomposition (SVD) of $A$ :

$$
A=U \Sigma V^{T} \quad \Rightarrow \quad A^{+}=V \Sigma^{-1} U^{T},
$$

where only nonzero singular values corresponding to observable modes are inverted. More formally, partition the matrices of the SVD as

$$
\begin{gathered}
A=\left[\begin{array}{llll}
U_{h} & U_{f} & U_{g} & U_{\mathrm{np}}
\end{array}\right]\left[\begin{array}{ccc}
\Sigma_{h} & 0 & 0 \\
0 & \Sigma_{f} & 0 \\
0 & 0 & \Sigma_{g} \\
0 & 0 & 0
\end{array}\right]\left[\begin{array}{c}
V_{h}^{T} \\
V_{f}^{T} \\
V_{g}^{T}
\end{array}\right] \\
=U_{h} \Sigma_{h} V_{h}^{T}+U_{f} \Sigma_{f} V_{f}
\end{gathered}
$$

where $(\cdot)_{f}$ refers to focus mode, $(\cdot)_{g}$ refers to the unobservable global piston, tip, and tilt (that is, $\Sigma_{g}=$ $\left.0_{3 \times 3}\right), U_{\mathrm{np}}$ is a basis in sensor space for the nonphysical degrees of freedom of the overdetermined sensor array, and $(\cdot)_{h}$ refers to the remaining degrees of freedom that are observable through the height sensitivity of the interaction matrix. Denote the smallest nonzero singular value of $A$ as $\sigma_{\min }(A)$; as noted earlier this is $\Sigma_{f}$ for nonzero but realistically small dihedral sensitivity. Because the height sensitivity $A_{h}$ does not affect focus mode, and the dihedral sensitivity $A_{\theta}$ to first order does not affect the remaining degrees of freedom, the two terms in Eq. (8) are approximately equal to $A_{h}$ and $A_{\theta}$, respectively. This is critical to understanding the sensitivity to errors or variation in the ratio of dihedral and height sensitivity, $L_{\text {eff }}$.

Using the partitioned $A$-matrix SVD, the pseudoinverse is

$$
A^{+}=V_{h} \Sigma_{h}^{-1} U_{h}^{T}+V_{f} \Sigma_{f}^{-1} U_{f}^{T} .
$$

If the pseudoinverse is calculated from the exact $A$ matrix, then $A^{+} A=V_{o} V_{o}^{T}=I-V_{g} V_{g}^{T}$, where $V_{o}=$
$\left[V_{h} V_{f}\right]$ is a basis for the observable subspace, and $V_{g}$ is a basis for the unobservable global modes. In general, however, the actual $A$ matrix (denoted $A^{\prime}$ ) differs slightly from that used in computing the pseudoinverse, either due to uncertainty, temporal variation (including sensor gain dependence on the intersegment gap), or intentional approximation.

Control of AO systems [5] also involves an estimation problem $\hat{x}=A^{+} y$ with sensor-actuator interaction as in Eq. (1). For a single-conjugate AO system with Shack-Hartmann wavefront sensors, the measurements $y$ are the residual (postcorrection) wavefront slopes and $x$ is the residual atmospheric phase at the deformable mirror actuator locations. There is no analog to the dihedral sensitivity present in the segmented-mirror control problem, and thus no distinction between $(\cdot)_{h}$ and $(\cdot)_{f}$ in the SVD of Eq. (7); otherwise, the interaction matrix properties are similar to the segmented-mirror control problem and noted in Section 5 .

\section{B. Sources of Error}

The following sources of error in the $A$ matrix are considered:

a. Errors in actuator gain.

b. Errors in sensor gain giving an overall scale error.

c. Errors in the ratio of sensor dihedral to height sensitivity.

d. Errors due to geometry.

The third source is specific to segmented-mirror control. These errors can be represented mathematically as

$$
\begin{gathered}
A_{i j}^{\prime}=A_{i j} \times\left(1+\delta_{j}\right), \\
A_{i j}^{\prime}=A_{i j} \times\left(1+\delta_{i}\right), \\
A_{i j}^{\prime}=\left(A_{h}\right)_{i j}+\left(A_{\theta}\right)_{i j} \times\left(1+\delta_{i}\right), \\
A_{i j}^{\prime}=A_{i j}+\delta_{i j} .
\end{gathered}
$$

Note that the gain errors in Eqs. (10)-(12) are defined to be relative, while the geometry error in Eq. (13) is an absolute variation in the elements of $A$. With $A$ dimensionless, the elements of $A$ are of order unity, and hence this choice is largely arbitrary but simplifies the analysis. The effect of additional structure on the geometry error in Eq. (13) is discussed in Subsection 3.B.

The next section provides an analytical bound $b$ on the magnitude of the allowable errors, so that stability is guaranteed for all errors satisfying $\left|\delta_{i}\right|<b$ (or $\left|\delta_{i j}\right|<b$ ) for some $b$ that will depend on the type of uncertainty, the problem, and problem-specific 
parameters such as segmentation geometry and dihedral sensitivity. The bound is both necessary and sufficient (not conservative) if there exists some set of uncertainties $\delta_{i}$ with $\left|\delta_{i}\right| \leq b$ for which the system is marginally stable. This does not imply that if the uncertainty exceeds the bound that instability is certain, simply that it is possible.

Note that if the errors are not bounded but have, e.g., a Gaussian probability distribution, then any nonzero uncertainty amplitude will result in a nonzero probability of instability. This requires Monte Carlo analysis to predict; however, even in this case, the following analysis is useful to understand the sensitivity.

\section{Analysis}

\section{A. Small Gain Analysis}

The block diagrams in Fig. 4 illustrate the structure of the uncertainty for the four cases listed in the previous section. The small gain theorem is first used to compute an analytical bound on the maximum allowable norm-bounded error. This may be conservative, since it does not account for the structure within the uncertainty. However, the conservative bound provides insight, and is sufficient to understand what types of errors are or are not a problem for control. Furthermore, for all of the gain errors [Figs. 4(a), 4(b), and 4(c)], it is shown that the bound obtained from the small gain theorem is both necessary and sufficient. Additional steps are required to estimate a nonconservative bound for geometry errors in $A$, using structured singular values ( $\mu$ analysis) [9].

Note that for each of the gain errors [Figs. 4(a), 4(b), and 4(c)], the block $\Delta$ in Fig. 4 should be constrained to be a real diagonal matrix, whereas small gain analysis will only constrain the matrix norm (maximum singular value). For the geometry errors in $(d)$, the block $\Delta$ should be constrained to be zero where the matrix $A$ is zero. The relationship between a bound on the singular values of an otherwise unconstrained matrix $\Delta$ and the desired bound $b$ on the nonzero elements of $A$ is not obvious.

Any dynamics can in principle be captured in the controller $K(s)$; however, the effects of dynamics on the control problem are described elsewhere [11] and aside from comments in Subsection 3.C are neglected here for simplicity. For simplicity, assume that the control is identical for each degree of freedom, so $K(s)=k(s) I$ for scalar $k(s)$; this is true at existing segmented-mirror telescopes and many existing AO systems. The effect of more complex control structures is noted in Subsection 3.C. Define the scalar complementary sensitivity

$$
T(s)=\frac{k(s)}{1+k(s)}
$$

Each block diagram in Fig. 4 can be transformed into a feedback loop between uncertainty $\Delta$ and a system $T(s) M$, where in order in the figure,
a. $M=A^{+} A$,
b. $M=A A^{+}$,
c. $M=A_{\theta} A^{+}$, and
d. $M=A^{+}$.

Note that to transform the feedback loop into $T(s) M$ with $T(s)$ scalar there is one non-obvious step involving the unobservable subspace. For all but case (b), loop analysis gives matrix-valued complementary sensitivity $\tilde{T}(s)=\left(I+k(s) A^{+} A\right)^{-1} k(s)$. Using the SVD of $A$ to change the basis, then the scalar $T(s)$ given in Eq. (14) is valid only on the observable subspace, spanned by $V_{o}$. However, since $M$ is zero on the unobservable subspace, $M=V_{o} V_{o}^{T} M$ and thus (a)

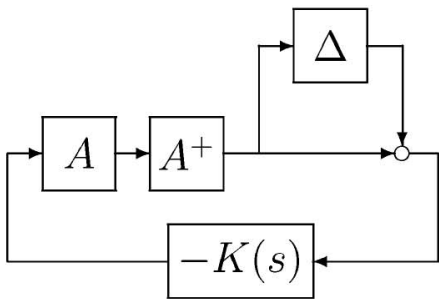

(c)

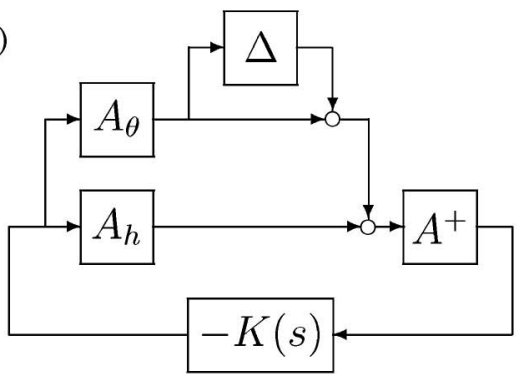

(b)

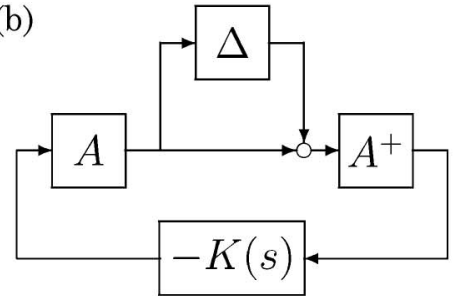

(d)

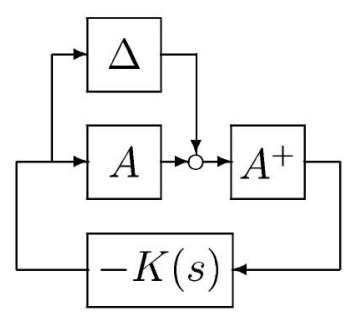

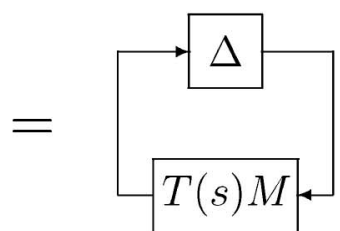

Fig. 4. Schematic of uncertainty $\Delta$ in interaction matrix $A$ with estimator $A^{+}$and control $K(s)$ : (a) uncertainty in actuator gain, (b) in sensor gain, (c) in dihedral gain, and (d) in matrix entries. In each case, the block diagram can be transformed into the form on the right, with $T(s) M$ given in the text. 
$\tilde{T}(s) M=\tilde{T}(s) V_{o} V_{o}^{T} M=T(s) V_{o} V_{o}^{T} M=T(s) M . \quad$ For case (b) the analysis is similar except that $T$ involves $A A^{+}$, and so the observable sensor subspace $U_{o}=$ $\left[U_{h} U_{f}\right]$ is used rather than the observable actuator subspace $V_{o}$.

The small gain theorem (e.g., $[7,8]$ ) guarantees that the feedback system on the right-hand side of Fig. $\underline{4}$ is stable for all $\Delta$ such that

$$
\|\Delta\|_{\infty}<\|M T\|_{\infty}^{-1}
$$

where here the infinity-norm $\|M T\|_{\infty}=\sigma_{\max }(M)$ $\sup _{\omega}(T)$, which is the largest singular value of the matrix $M$ multiplied by the supremum over frequency of the scalar transfer function $T(s)$.

Note that $\|T\|_{\infty} \geq 1$ for any nonzero integral control gain (as is used in any existing active or adaptive optics systems). If the system dynamics can be ignored (if the bandwidth is significantly lower than any resonant frequencies) and there is negligible time delay, then $\|T\|_{\infty} \simeq 1$. For realistic but robust control loops with nonzero latency and structural dynamics, then $\|T\|_{\infty}<2$ is reasonable. This implies that changing the bandwidth of the control loop is at most a factor of 2 effect on the required accuracy.

Thus, from the small gain theorem, then in the limit of small bandwidth $\left(\|T\|_{\infty}=1\right)$, stability is guaranteed in the four cases for

$$
\begin{array}{ll}
\text { a. } & \|\Delta\|_{\infty}<1, \\
\text { b. }\|\Delta\|_{\infty}<1, \\
\text { c. }\|\Delta\|_{\infty}<1 / \sigma_{\max }\left(A_{\theta} A^{+}\right), \text {and } \\
\text { d. }\|\Delta\|_{\infty}<\sigma_{\min }(A) .
\end{array}
$$

For higher control bandwidth with nonzero latency, the required bound is tighter by a factor of $\|T\|_{\infty}$.

In the first case, an actuator gain error clearly implies only that the corresponding position will have a bandwidth error roughly equal to the gain error (that is, the gain error uses up some of the gain margin designed into the system; see Subsection 3.C). This intuition is confirmed by the analysis: the system is quite robust to this type of uncertainty (although there will be performance penalties if the errors approach the stability boundary).

The second case, with random multiplicative errors in the measurement $y$, corresponds to uncertainty in the sensor gain. The intuition here is similar to the case of actuator gain errors. The component of the sensor reading that is in the physical subspace will be driven more or less rapidly to its desired setpoint than its neighbors, but the solution will still converge even with large (but less than unity) variations in sensor gain. For both the sensor and the actuator gain cases it is straightforward from construction (e.g., choose $\Delta=\alpha I$ ) to see that the bound obtained from the small gain theorem is both necessary and sufficient. That is, there exists an uncertainty satisfying $\left|\delta_{i}\right| \leq 1$ for which the closed-loop system is not asymptotically stable.
The third case corresponds to uncertainty in the dihedral sensitivity only, and is specific to the segmented-mirror control problem. Here, note that if we transform into the SVD basis of $A$, then for small $L_{\text {eff }} / a$ the dihedral sensitivity $A_{\theta}$ primarily affects focus mode, while the height sensitivity $A_{h}$ affects only orthogonal modes. From Eqs. (8) and (9) and the discussion surrounding them, it follows that

$$
A_{\theta} A^{+} \simeq U_{f} U_{f}^{T}
$$

and $\sigma_{\max }\left(A_{\theta} A^{+}\right) \simeq 1$ follows immediately, with the inexact equality because the dihedral sensitivity does slightly couple into the other modes. (It is easily verified from computation that the approximation is valid for realistic choices of $L_{\text {eff }} / a$ ). Thus the system is quite robust to this type of uncertainty also. Again, choosing $\Delta=\alpha I$ is sufficient to illustrate that this robustness bound is both necessary and sufficient.

The final case, with random errors directly in the elements of $A$, corresponds to uncertainty in geometry and requires that these errors be smaller than the smallest nonzero singular value of $A$. This can be quite small; e.g., with the 492-segment TMT geometry and $L_{\text {eff }}=10 \mathrm{~mm}$, then $\sigma_{\min }(A)$ is of order $6 \times$ $10^{-4}$. Thus the small gain result is sufficient to illustrate that the control system is very sensitive to uncertainty of this type. However, it is not immediately apparent either how conservative this stability bound is or how to relate a bound on the maximum singular value of $\Delta$ to bounds on $\delta_{i j}$ in Eq. (13). For this type of uncertainty, additional analysis is required, given in Subsection 3.B.

The above analysis does not prove robustness to simultaneous errors, e.g., in both sensor and actuator gains. Structured singular values ( $\mu$ analysis) could be used to analyze this case; however, the analysis is not straightforward, and it is easier to directly consider the effects. With uncertainty $\Delta_{1}$ on sensor gain and $\Delta_{2}$ on actuator gain, instability occurs if

$$
\operatorname{det}\left[I-T(s) A^{+}\left(I+\Delta_{1}\right) A\left(I+\Delta_{2}\right)\right]=0
$$

for some $\Delta_{1}$ and $\Delta_{2}$. The worst case uncertainty as before is $\Delta_{1}=-\alpha_{1} I, \Delta_{2}=-\alpha_{2} I$. Each uncertainty independently affects the gain margin of the system, and so the combined effect is multiplicative rather than additive, and the stability boundary remains as before.

A similar analysis can be used to prove robustness to other combinations of simultaneous gain errors (including the sensor dihedral gain). Thus, from a design perspective, it is only the geometry errors that are a potentially significant concern.

\section{B. Geometry Errors}

Now return to the geometry errors for which the small gain analysis illustrates that the control system is sensitive to the errors but does not immediately lead to a useful bound on the specific structure of the uncertainty. A tighter bound on the 
allowable uncertainty can be obtained using structured singular values. First, use Eq. (3) to rewrite the error in Fig. 4 so that the uncertainty block is diagonal with nonzero elements corresponding to the nonzero elements of $A$ :

$$
\Delta=\mathcal{P} \underline{\Delta} \mathcal{R} .
$$

With the diagonal uncertainty $\Delta$, the feedback loop on the right-hand side of Fig. $\underline{4}$ is between $\underline{\Delta}$ and $M=\mathcal{R} A^{+} \mathcal{P}$.

Henceforth, drop the underbar notation, so that the uncertainty $\Delta$ refers to the real, diagonal uncertainty with bound on each diagonal element of $\left|\delta_{i i}\right|<b$. The estimate of the largest uncertainty bound $b=\mu^{-1}$ for which the system is guaranteed to be stable for all $\Delta$ of this form can be obtained from the bounds [[9], Eq. (3.10) and Thm. 6.4]

$$
\max _{Q \in \mathcal{Q}} \rho(Q M)=\mu \leq \sigma_{\max }(M),
$$

where the maximization on the left-hand side is over $Q$ with the same structure as $\Delta$ (diagonal), with diagonal entries $q_{i i}= \pm 1$. With $Q^{*}$ maximizing $\rho(Q M)$, then the smallest uncertainty that results in instability is $\Delta=\mu^{-1} Q^{*}$. Although $M \in \mathbb{R}^{q m \times q m}$ is large, both the spectral radius $\rho(M)$ and maximum singular value $\bar{\sigma}(M)=\left[\rho\left(M^{T} M\right)\right]^{1 / 2}$ are easily obtained through power methods without actually constructing $M$. Iterative methods exist to search over $Q$ but converge only to local maxima so that in general $\mu$ can only be bounded above and below. The bound $\mu$ depends on both $M$ and the structure of the uncertainty $\Delta$.

If every nonzero element of $A$ has independent uncertainty, then the diagonal elements of $Q$ in Eq. (18) can be chosen independently. If there is additional structure on the uncertainty, then the identical additional structure is placed on $Q$; this clearly results in a smaller value for $\mu$ and hence larger uncertainty that can be tolerated, as expected. As an example, consider $A=A_{0}+\sum_{i=1}^{p} d_{i} A_{i}$ for uncertain but bounded scalars $d_{i}$. This structure captures, e.g., AO misregistration errors or specific shifts in $A$ due to a specific set of scalar uncertainties. The corresponding uncertainty has structure

$$
\Delta=\left[\begin{array}{ccc}
d_{1} I_{1} & 0 & \cdots \\
0 & d_{2} I_{2} & \\
\vdots & & \ddots
\end{array}\right]
$$

where each identity block $I_{i}$ has dimension equal to the rank of $A_{i}$. The matrix $Q$ in Eq. (18) has the same structure, and the maximization is thus over the same number of variables as there are uncertain parameters. If there is a single uncertain parameter (e.g., a single axis misregistration), then $M=A_{0}^{+} A_{1}$, $Q= \pm I$, and the solution reduces to the obvious $\mu=\rho\left(A_{0}^{+} A_{1}\right)$.

\section{Dynamics}

The analytical bounds computed above guarantee stability in the limit of small control bandwidth. Conclusions for the more general case are easily understood, since as noted earlier the bound on uncertainty required to guarantee stability scales inversely with $\|T(s)\|_{\infty}$, typically a factor of 2 or so tighter. For an uncertainty that is sufficiently small so as to guarantee stability, the uncertainty will still contribute to a reduction in stability margin. Thus, to allow a reasonable allowance for other sources of uncertainty, the allowable bound on interaction-matrix errors should be a factor of a few smaller than the stability limit.

Spatial filtering (as in [6]) or modal control (common in AO, also suggested for segmented-mirror control [11]) can be incorporated by substituting $B \neq A^{+}$ in the definition of $M$ and computing the corresponding bounds from small gain or $\mu$ analysis. However, as long as the control still has nonzero integral gain in all observable directions, the low bandwidth robustness limit will not be changed. What will change is that the maximum complementary sensitivity will be closer to unity for certain directions, and thus a higher uncertainty may be tolerated, by of order a factor of 2 .

\section{Segmented-Mirror Example}

Since any interaction-matrix based estimation problem is robust to uncertainty in actuator or sensor gain, only the geometry errors are explored in example. The interaction matrix of an idealized flat mirror tiled with regular hexagons is constructed as described in [3]. For the 492-segment mirror shown in Fig. 1 with $L_{\text {eff }} / a=0.014$, the procedure in Subsection $\overline{3}$. B leads to

$$
1.2 \times 10^{4} \leq \mu \leq 1.4 \times 10^{4} .
$$

That is, an uncertainty in the elements of $A$ can be constructed where each element of $A$ is perturbed by no more than $\pm 8.4 \times 10^{-5}$, and the resulting closed-loop control system will be unstable. If all perturbations in $A$ can be guaranteed to be less than $\pm 7.1 \times 10^{-5}$, then stability is guaranteed. These are clearly tight requirements; e.g., for mirror segments with dimensions of order $1 \mathrm{~m}$, then sensor and segment installation tolerances will need to be of order $70 \mu \mathrm{m}$ to guarantee stability.

The corresponding numbers for Keck (36 segments, $\left.L_{\text {eff }}=55 \mathrm{~mm}, a=0.9 \mathrm{~m}\right)$ are

$$
204 \leq \mu \leq 245 .
$$

As illustrated in Fig. 5, $\mu$ scales linearly with the number of segments; this is why interaction-matrix robustness was not an issue at existing observatories but could be an issue at future ones. The maximum tolerable uncertainty $\mu^{-1}$ scales linearly with $L_{\text {eff }} / a$. The worst-case pattern of uncertainty leads to instability of focus mode. If focus mode is not 
controlled (or $L_{\text {eff }}=0$ ), then the worst-case pattern of uncertainty leads to instability of astigmatism, and roughly a factor of 10 larger errors are tolerable than with $L_{\text {eff }} / a=0.014$.

Note that this analysis considers all possible bounded variations in the nonzero elements of the $A$ matrix. The specific pattern that leads most rapidly toward instability is physically possible, but a randomly selected variation in the $A$ matrix is extremely unlikely to be as destabilizing as the worstcase bounds above. The extent of destabilization for any given perturbation $\Delta$ can be evaluated by computing the spectral radius $\rho(\Delta M)$. It is thus straightforward to estimate the probability distribution and compute a more relaxed bound that, while not sufficient to guarantee stability, provides any specified level of confidence.

Figure 6 illustrates the potential destabilization for both the geometry-type errors in the interaction matrix, and for gain errors (the same for actuator, sensor, or dihedral sensitivity), again using the 492segment mirror array with $L_{\text {eff }} / a=0.014$. The worst-case nonzero eigenvalue of $A^{+} A^{\prime}$ is plotted (equivalent to $1-\rho(\Delta M)$; the three eigenvalues at zero corresponding to unobservable global piston, tip, and tilt, are not included.) For the gain errors, only the guaranteed bound is plotted, since any highconfidence probabilistic bound is not significantly different. For geometry errors, for which the worstcase destabilizing uncertainty has a very specific and unlikely structure, then both the guaranteed bound and the 95\% confidence bounds are shown, where in the latter case the uncertainty is sampled from a uniform distribution.

If there is additional structure on the uncertainty, the same basic analysis technique of transforming the uncertainty into a diagonal structure and searching over diagonal $Q$ to bound $\mu$ using Eq. (18) can be

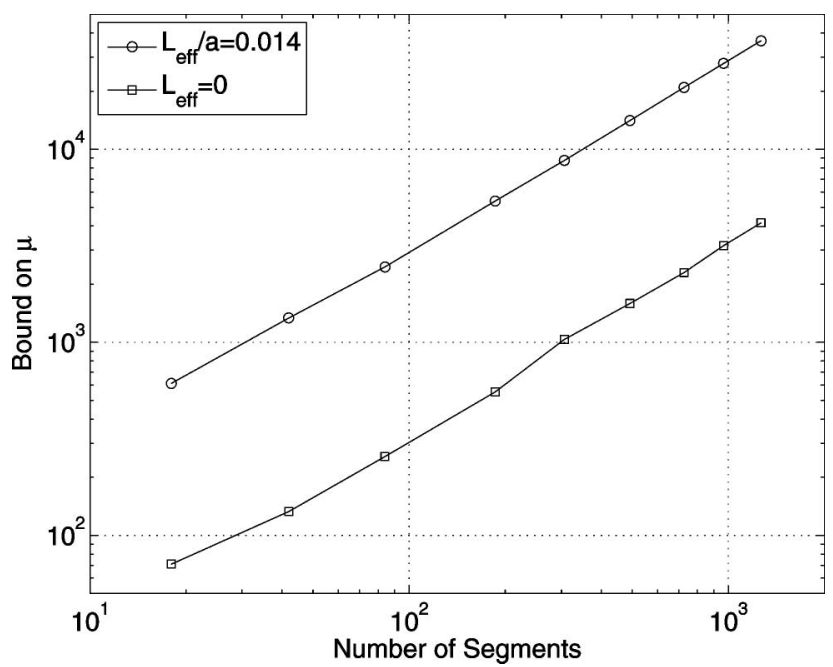

Fig. 5. Inverse stability bound $\mu$ as a function of number of segments in the mirror, for constant $L_{\text {eff }} / a$ and for $L_{\text {eff }}=0$. In the latter case, focus mode is not controlled; in the former, $\mu$ scales inversely with $L_{\text {eff }} / a$. Only the upper bound on $\mu$ is shown; the lower bound is roughly $15 \%$ lower. Values for $t / a$ and $f / a$ as in Fig. $\underline{2}$.

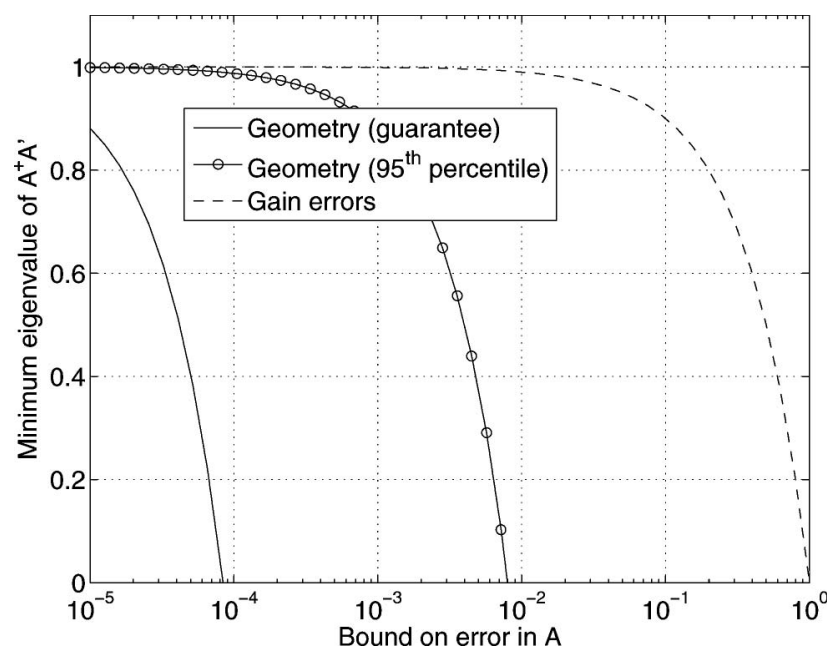

Fig. 6. Worst-case (nonzero) eigenvalue of $A^{+} A^{\prime}$ evaluated as a function of uncertainty magnitude, for a 492-segment primary mirror with $L_{\text {eff }} / a=0.014$. For geometry errors, both the analytical bound and the results of Monte Carlo analysis to estimate the 95\% confidence bounds are shown. Instability will occur for any nonzero control bandwidth if the minimum eigenvalue drops below zero.

used to explore the sensitivity to any desired parameters, e.g., uncertainty in actuator or sensor locations. Clearly, as the description of uncertainty is further constrained, the tolerable bounds $\mu^{-1}$ will increase.

\section{Adaptive Optics}

As with segmented-mirror control, AO control will be robust to uncertainty in either sensor or actuator gain, but potentially sensitive to "geometry"-type errors in which every nonzero element of the interaction matrix can vary independently.

With a Fried geometry as shown in Fig. 7, each sensor is (ideally) influenced by $q=4$ adjacent actuators, and there are two unobservable modes, corresponding to overall wavefront piston and "waffle" mode (alternating pattern of positive and negative displacement at actuator locations). The decomposition of the $A$ matrix in Eq. (3) can also be constructed, where now the diagonal matrix $\underline{A} \in \mathbb{R}^{q m \times q m}$ with $q=$ 4. If one wanted to characterize robustness to nonzero deformable-mirror influence matrix beyond the neighboring slopes, a larger matrix $\underline{A}$ could be defined; this would also be required for misregistration errors.

To explore geometry-type errors quantitatively, the $A$ matrix is constructed for a Fried geometry, with slope measurements obtained as the phase difference between adjacent actuator locations (without normalizing by the distance between actuators) so that all nonzero elements of $A$ are \pm 0.5 . Uncertainty bounds can again be obtained using Eq. (18). For example, with the $17 \times 17$ actuator grid shown in Fig. 7, giving $n=241$ actuators and $m=416$ sensors (the Palomar Observatory geometry), then

$13 \leq \mu \leq 25$. 


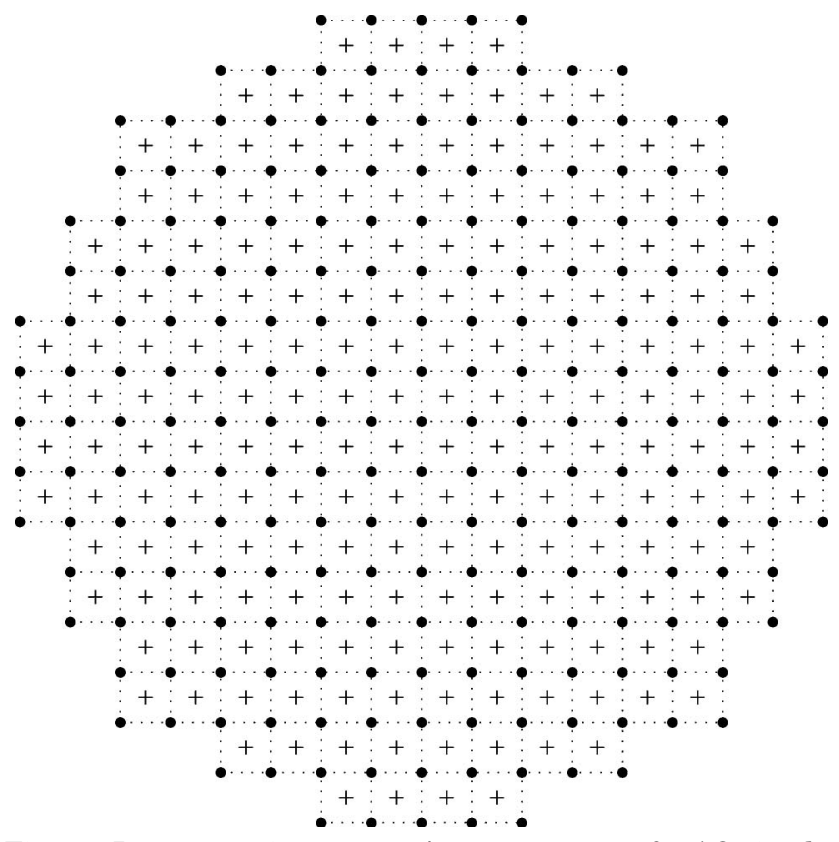

Fig. 7. Representative actuator/sensor geometry for AO simulations (with the central obscuration ignored for simplicity) where "•" represent actuators and "+" a two-axis tip/tilt measurement.

For a much larger future AO system (as an example, choosing circularized $65 \times 65$ actuator grid with $n=$ 3313 actuators and $m=6398$ sensors), then

$$
50 \leq \mu \leq 98 .
$$

The bound $\mu$ here increases only with the square root of the number of actuators (linear in the number of actuators across the aperture), as illustrated in Fig. 8 .

Once again, the specific structure of uncertainty that most rapidly leads to uncertainty is not likely. Comparing these bounds on $\mu$ with the corresponding bounds for the segmented-mirror control problem, it is clear that arbitrary interaction-matrix uncertainty robustness is a much less significant concern for (single-conjugate) adaptive optics than it is for segmented-mirror control, even for future large AO problems.

Structured uncertainty such as misregistration could be analyzed for a specific problem using Eqs. (18) and (19). For single-axis (single degree of freedom in $\Delta$ ) the result is identical to that obtained by direct computation of $\rho\left(A_{0}^{+} A_{1}\right)$, where $A_{1}$ describes the perturbation.

\section{Conclusions}

Both active and adaptive optics systems for future large optical telescopes involve many more actuators and sensors than at existing facilities. For control of highly segmented primary mirrors, feedback is provided by sensors that measure relative motion between neighboring segments. The sensor response is related to segment displacement through an interaction matrix. The position of each mirror segment is

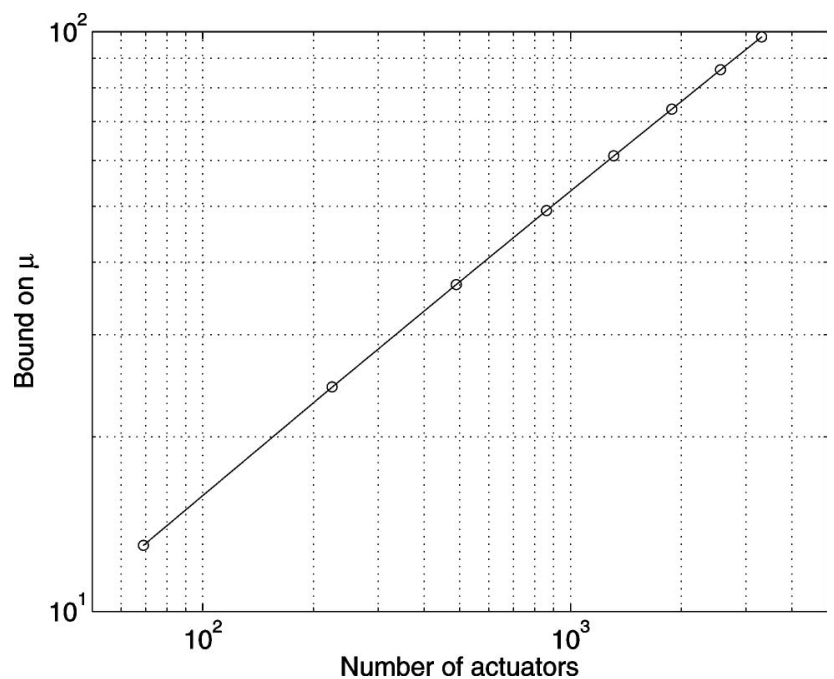

Fig. 8. Inverse stability bound $\mu$ as a function of number of deformable mirror actuators for single-conjugate $\mathrm{AO}$.

then estimated, typically using the pseudoinverse of this matrix. For a telescope with many segments, the interaction matrix is ill conditioned, and small errors or uncertainty in the elements of the matrix can lead to large estimation errors and potentially instability. AO control involves a similar interaction matrix; however, the matrix is better conditioned.

Small gain and structured singular value analysis tools are used to compute a bound on the magnitude of allowable uncertainty for which the closed-loop system is guaranteed to be stable. This illustrates that the control is quite robust to uncertainty in actuator or sensor gain. For segmented-mirror sensors that respond to both intersegment height and dihedral angle, the control is also robust to the relative sensitivity between these two parameters. However, very small errors in the geometry between actuators and sensors can lead to very large estimation errors and control instability.

Geometry errors in segmented-mirror control include, e.g., the effects of sensor installation errors. However, sensor/actuator nonlinearities, thermal dependencies, sensor output dependence on intersegment gap, or slight changes in frequency response between different actuators or sensors all manifest as errors in actuator or sensor gain, and the control system is quite robust to these errors.

Examples are given for both segmented-mirror and $\mathrm{AO}$ control. In the former case, the sensitivity to errors grows linearly with the number of segments in the mirror. If focus mode of the mirror array is controlled, the sensitivity to errors is inversely proportional to the dihedral sensitivity of the segments. These two factors make robustness to interactionmatrix uncertainty much more sensitive than at existing telescopes, and may require that the interaction matrix be accurately measured after segment installation in order to provide robustness to segment and sensor installation tolerances. For AO, the 
sensitivity to errors increases only with the square root of the number of actuators.

The approach presented can be applied to any specific structure in the uncertainty, e.g., for AO misregistration or for uncertainty or variation in specific geometric parameters for segmented-mirror control. Additional structure leads to larger bounds on the tolerable magnitude of uncertainty.

This work was supported by the TMT Project. The TMT Project gratefully acknowledges the support of the TMT partner institutions. They are the Association of Canadian Universities for Research in Astronomy (ACURA), the California Institute of Technology, and the University of California. This work was supported as well by the Gordon and Betty Moore Foundation, the Canada Foundation for Innovation, the Ontario Ministry of Research and Innovation, the National Research Council of Canada, the Natural Sciences and Engineering Research Council of Canada, the British Columbia Knowledge Development Fund, the Association of Universities for Research in Astronomy (AURA) and the U.S. National Science Foundation (NSF).

\section{References}

1. J. Nelson and G. H. Sanders, "The status of the Thirty Meter Telescope project," Proc. SPIE 7012, 70121A (2008).

2. R. Gilmozzi and J. Spyromilio, "The $42 \mathrm{~m}$ European ELT: status," 7012, 701219 (2008).
3. G. Chanan, D. G. MacMartin, J. Nelson, and T. Mast, "Control and alignment of segmented-mirror telescopes: matrices, modes, and error propagation," Appl. Opt. 43, 1223-1232 (2004).

4. R. C. Jared, A. A. Arthur, S. Andreae, A. Biocca, R. W. Cohen, J. M. Fuertes, J. Franck, G. Gabor, J. Llacer, T. Mast, J. Meng, T. Merrick, R. Minor, J. Nelson, M. Orayani, P. Salz, B. Schaefer, and C. Witebsky, "The W. M. Keck Telescope segmented primary mirror active control system," Proc. SPIE 1236, 996-1008 (1990).

5. J. W. Hardy, Adaptive Optics for Astronomical Telescopes, Oxford Series on Optical and Imaging Sciences (Oxford University Press, 1998).

6. T. J. Brennan, "Stability margin loss due to wavefront sensor misregistration: amelioration with spatial filtering techniques," Proc. SPIE 4376, 88-98 (2001).

7. K. J. Åström and R. M. Murray, Feedback Systems: An Introduction for Scientists and Engineers (Princeton University Press, 2008).

8. J. C. Doyle, B. A. Francis, and A. R. Tannenbaum, Feedback Control Theory (MacMillan, 1992).

9. A. Packard and J. Doyle, "The complex structured singular value," Automatica 29, 71-109 (1993).

10. D. G. MacMartin and G. Chanan, "Measurement accuracy in control of segmented-mirror telescopes," Appl. Opt. 43, 608-615 (2004).

11. D. G. MacMynowski, P. M. Thompson, and M. J. Sirota, "Control of many coupled oscillators and application to segmented-mirror telescopes," presented at the AIAA Guidance, Navigation and Control Conference, Honolulu, Hawaii, 18-21 August 2008. 